\title{
MULTISCALE SCATTERING IN NONLINEAR KERR-TYPE MEDIA
}

\author{
Roland Maier*, Barbara Verfürth ${ }^{\dagger}$ \\ * Department of Mathematical Sciences \\ Chalmers University of Technology and University of Gothenburg \\ Chalmers Tvärgata 3, 41296 Göteborg, Sweden \\ e-mail: roland.maier@chalmers.se \\ $\dagger$ Institut für Angewandte und Numerische Mathematik \\ Karlsruher Institut für Technologie \\ Englerstr. 2, 76131 Karlsruhe \\ e-mail: barbara.verfuerth@kit.edu
}

\begin{abstract}
Wave propagation in heterogeneous and nonlinear media has arisen growing interest in the last years since corresponding materials can lead to unusual and interesting effects and therefore come with a wide range of applications. An important example for such materials are Kerr-type media, where the intensity of a wave directly influences the refractive index. In the time-harmonic regime, this effect can be modeled with the nonlinear Helmholtz equation

$$
-\operatorname{div} A \nabla u-k^{2} n\left(1+\varepsilon \mathbb{1}_{D_{\varepsilon}}|u|^{2}\right) u=f,
$$

where $D_{\varepsilon}$ is the subdomain where the nonlinear Kerr-type medium is active, $A, n$, and $\varepsilon$ are material coefficients and $k$ is the wave number. In this contribution, the coefficients $A, n, \varepsilon$ may vary on small spatial scales, such that the numerical approximation of corresponding solutions can be a delicate task.

To deal with microscopic coefficients without the need for global fine-scale computations, multiscale methods can be applied. One such method is the Localized Orthogonal Decomposition method (LOD), which works under minimal structural assumptions. The approach was proposed in [MP14] and refined in [HP13] for an elliptic model problem and constructs appropriate coarsescale spaces that take into account problem-dependent information. In this talk, which is based on [MV20], an iterative and adaptive construction of approximation spaces based on the LOD is presented. The general idea is to combine ideas of [WZ18] on iterative finite element approximations with the above-mentioned multiscale approach. That is, in each iteration step, a new coarse-scale solution is constructed based on the solution of the previous step. To avoid costly re-computations, an error indicator is used to locally decide in each step whether to update the approximation space. For sufficiently small tolerance employed in this decision, an a priori error estimate can be shown which is of optimal order in the mesh size - independent of the possible low regularity of the exact solution. These results are also illustrated by numerical experiments.
\end{abstract}

\section{REFERENCES}

[HP13] P. Henning and D. Peterseim. Oversampling for the multiscale finite element method. Multiscale Model. Simul., 11(4):1149-1175, 2013.

[MP14] A. Målqvist and D. Peterseim. Localization of elliptic multiscale problems. Math. Comp., 83(290):25832603, 2014.

[MV20] R. Maier and B. Verfürth. Multiscale scattering in nonlinear Kerr-type media. ArXiv Preprint, 2011.09168, 2020.

[WZ18] H. Wu and J. Zou. Finite element method and its analysis for a nonlinear Helmholtz equation with high wave numbers. SIAM J. Numer. Anal., 56(3):1338-1359, 2018. 